\title{
The general mixed nonlinear Schrödinger equation: Darboux transformation, rogue wave solutions, and modulation instability
}

\author{
Wenbo Li , Chunyan Xue* and Lili Sun
}

"Correspondence:

liwnebo002005@sina.com; xue-chunyan@126.com

School of Applied Science, Beijing Information Science and

Technology University, Beijing, 100192, China

\section{包 Springer}

\begin{abstract}
In this paper, the Darboux transformation method has been successfully applied to a general mixed nonlinear Schrödinger equation and some rogue wave solutions are proposed. First of all, the determinant representation of an $n$-fold DT is given explicitly. Then starting with a periodic seed solution, we obtain some rogue wave solutions of the general mixed nonlinear Schrödinger equation through iteration of a generalized DT. Second, the three-dimensional images and density profiles of the rogue waves are plotted to show the structures of these rogue wave solutions. Finally, we give evidence for the connection between the occurrence of rogue wave solutions and the modulation instability.
\end{abstract}

Keywords: Darboux transformation; rogue wave solution; general mixed nonlinear Schrödinger equation; Lax pair; modulation instability

\section{Introduction}

In the past decades, there are extensive advancements in the field of nonlinear integrable systems. How to get the exact soliton solutions of these integrable systems has become one of the important research topics in theory and practical applications. Quite a few approaches for finding exact solutions of such nonlinear systems are well established, such as the inverse scattering method [1], the Darboux transformation method [2,3], the Bäcklund transformation [4-6], the Riemann-Hilbert formulation [7-9], the Hirota bilinear method [10,11], Lie group analysis [12], the similarity transformation method [13-16], and the homotopy analysis method [17-20], F-expansion method [21] and so on [22, 23]. Among these approaches, the DT is well known to be a powerful method for finding exact solutions of integrable systems [2].

In recent years, more researchers have begun to pay attention to rouge waves (RWs), which has been introduced and become an interesting objective in some fields, such as optics [24-27], super fluids [28], Bose-Einstein condensates [29-31], and so on. RW is one terrible reason to generate large marine catastrophes, which is a presence that may always show up with special giant waves in a very short time, and there is no sign before it appears. For example, the giant waves of several tens of meters will suddenly appear in a relatively calm sea. In optics, the wave will appear as a very bright spot, which mathematical physicists call rogue waves [32]. But now, the study of the rogue waves is still only

(c) $2016 \mathrm{Li}$ et al. This article is distributed under the terms of the Creative Commons Attribution 4.0 International License (http://creativecommons.org/licenses/by/4.0/), which permits unrestricted use, distribution, and reproduction in any medium, provided you give appropriate credit to the original author(s) and the source, provide a link to the Creative Commons license, and indicate if changes were made. 
in its infancy. Mechanisms and the probability of its occurrence are not clear. Because observing of rogue waves on the ocean is very difficult and dangerous, unreliable and few records and observations are available, which led to a lack of research on the rogue wave scientific community recognizing the factual basis.

One of the models to describe the rogue waves is the nonlinear Schrödinger (NLS) equation,

$$
i q_{t}+\frac{1}{2} q_{x x}+|q|^{2} q=0
$$

which is an important integrable nonlinear wave equation. Recently, much work was done to study various NLS equations [33-36].

By DT [37], one can get its first-order and high-order rogue wave solutions [38]. Recently, more general rogue waves of the higher-order NLS equation have been calculated $[39,40]$ and the spatial-temporal structures have also been discovered and analyzed.

It is well known that there is an integrable mixed NLS equation [41],

$$
q_{t}-i q_{x x}+a_{1}\left(q^{*} q^{2}\right)_{x}+i b q^{*} q^{2}=0
$$

where $q$ represents a complex field envelop and the asterisk denotes complex conjugation, $a$ and $b$ are two nonnegative constants. The mixed NLS equation is used to model the propagations of the Alfvén waves in plasmas and the ultrashort light pulse in optics.

Motivated by the work of [41], we will consider the following general mixed NLS equation:

$$
\begin{aligned}
& i q_{t}+q_{x x}+i \beta\left(|q|^{2} q\right)_{x}+i \alpha q_{x}+|q|^{2} q+r q=0, \\
& -i p_{t}+p_{x x}-i \beta\left(|q|^{2} p\right)_{x}-i \alpha p_{x}+|q|^{2} p+r p=0
\end{aligned}
$$

where $\alpha, \beta$, and $r$ are real constants. Under the condition $q^{*}=p$, this equation can be simplified as

$$
i q_{t}+q_{x x}+i \beta\left(|q|^{2} q\right)_{x}+i \alpha q_{x}+|q|^{2} q+r q=0 .
$$

This equation can be taken as one version of the Wadati-Konno-Ichikawa (WKI) system [42], and the corresponding Lax pair is given based on the WKI spectral problem

$$
\begin{aligned}
& \Psi_{x}=U \Psi=\left(i \beta I \lambda^{2}+Q_{1} \lambda+Q_{0}\right) \Psi, \\
& \Psi_{t}=V \Psi=\left(-2 i \beta^{2} I \lambda^{4}+V_{3} \lambda^{3}+V_{2} \lambda^{2}+V_{1} \lambda+V_{0}\right) \Psi,
\end{aligned}
$$

with $U$ and $V$ being $2 \times 2$ matrices, and

$$
\begin{aligned}
& I=\left(\begin{array}{cc}
1 & 0 \\
0 & -1
\end{array}\right), \quad Q_{1}=\left(\begin{array}{cc}
\sqrt{2} & \left(\frac{\alpha \beta+\sqrt{2} \omega \beta}{2+\alpha \beta}-\beta\right) q \\
\left(-\beta-\frac{\sqrt{2} \omega \beta}{2}\right) p & -\sqrt{2}
\end{array}\right), \\
& Q_{0}=\left(\begin{array}{cc}
-\frac{1}{4} i \alpha & \frac{\sqrt{2}}{2} i q \\
\left(\frac{\sqrt{2}}{2} i+\frac{\sqrt{2}}{4} i \alpha \beta\right) p & \frac{1}{4} i \alpha
\end{array}\right), \quad V_{3}=\left(\begin{array}{cc}
-4 \sqrt{2} \beta & \frac{2\left(2 \beta^{2}-\sqrt{2} \beta^{2} \omega\right)}{2+\alpha \beta} q \\
\left(2 \beta^{2}+\sqrt{2} \beta^{2} \omega\right) p & 4 \sqrt{2} \beta
\end{array}\right),
\end{aligned}
$$




$$
\begin{aligned}
& V_{2}=\left(\begin{array}{cc}
-i \beta^{2} q p+4 i & \frac{\beta}{2+\alpha \beta} m_{2} q \\
\frac{\beta}{2} m_{1} p & i \beta^{2} q p-4 i
\end{array}\right), \quad V_{1}=\left(\begin{array}{cc}
-\sqrt{2} \beta p q & \frac{1}{2(2+\alpha \beta)}\left(m_{3} q_{x}+m_{5} q\right) \\
\frac{1}{4}\left(m_{4} p_{x}+m_{6} p\right) & \sqrt{2} \beta p q
\end{array}\right), \\
& V_{0}=\left(\begin{array}{cc}
\frac{1}{8} i \alpha^{2}+\frac{1}{4} i \alpha \beta q p+\frac{1}{2} i r+\frac{1}{2} i p q & \frac{\sqrt{2}}{2} q_{x}-m_{7} q \\
\left(\frac{\sqrt{2}}{2}+\frac{\sqrt{2}}{4} \alpha \beta\right) p_{x}-m_{8} p & -\frac{1}{8} i \alpha^{2}-\frac{1}{4} i \alpha \beta q p-\frac{1}{2} i r-\frac{1}{2} i q p
\end{array}\right),
\end{aligned}
$$

where

$$
\begin{aligned}
& m_{1}=-6 \sqrt{2} i-\sqrt{2} i \alpha \beta-4 i \omega, \quad m_{2}=6 \sqrt{2} i+\sqrt{2} i \alpha \beta-4 i \omega, \\
& m_{3}=4 i \beta-2 \sqrt{2} i \beta \omega, \quad m_{4}=4 i \beta+2 \sqrt{2} i \beta \omega, \\
& m_{5}=8+2 \alpha \beta-4 q p \beta^{2}+2 \sqrt{2} \beta^{2} \omega q p+\sqrt{2} \alpha \beta \omega, \\
& m_{6}=4 \beta q p+2 \sqrt{2} \omega q p-2 \alpha \beta+\sqrt{2} \omega \alpha \beta-8, \quad m_{7}=\frac{\sqrt{2}}{2} i \beta q p+\frac{\sqrt{2}}{4} i \alpha, \\
& m_{8}=\frac{\sqrt{2}}{4} i \alpha \beta^{2} q p+\frac{\sqrt{2}}{8} i \alpha^{2} \beta+\frac{\sqrt{2}}{4} i \alpha+\frac{\sqrt{2}}{2} i \beta q p, \quad \omega=\sqrt{-\alpha \beta} .
\end{aligned}
$$

Here the complex number $\lambda$ is the associated spectral parameter, and $\Psi$ is the eigenfunction associated with $\lambda$ of the system. Equations (3) and (4) can be obtained by the zerocurvature equation $U_{t}-V_{x}+[U, V]=0$ of (6a) and (6b). It is well known that some exact solutions of the mixed NLS equation (5) with $\alpha=0$ have been constructed via DT [43-46] and the Hirota method $[47,48]$.

The main aim of this paper is to construct the DT to derive the rogue wave solutions of mixed NLS equation (5) by using the generalized Darboux transformation, then analyze the rogue wave through their figures. In the end, we investigate modulation instability of the mixed NLS equation and point out the connection between RW and modulation instability.

\section{Darboux transformation}

Based on procedure of Darboux transformation for AKNS system in [49], we can construct a Darboux matrix $T$ to satisfy $\Psi^{[1]}=T \Psi$, and to present the determinant representation of the $n$-fold transformation.

Assume the Darboux matrix $T$ is the form of

$$
T_{1}=\left.T_{1}\right|_{\lambda=\lambda_{1}}=\left(\begin{array}{cc}
a_{1} & 0 \\
0 & d_{1}
\end{array}\right) \lambda+\left(\begin{array}{ll}
a_{0} & b_{0} \\
c_{0} & d_{0}
\end{array}\right),
$$

where $a_{1}, d_{1}, a_{0}, b_{0}, c_{0}, d_{0}$ are undetermined functions of $(x, t)$, which will be parameterized by the eigenfunction associated with $\lambda_{1}$ and the seed solution $(q, p)$ for equations (3) and (4).

We consider $n$ eigenfunctions $\Psi_{j}$ as

$$
\Psi_{j}=\left(\begin{array}{c}
\phi_{j} \\
\varphi_{j}
\end{array}\right)
$$

where $j=1,2, \ldots, \phi_{j}=\phi_{j}\left(x, t, \lambda_{j}\right), \varphi_{j}=\varphi_{j}\left(x, t, \lambda_{j}\right)$, which is the eigenfunction of the Lax pair equations (6a)-(6b) with seed solution $(q, p)$ and spectral parameters $\lambda_{j}$. Then we can get 
the Darboux matrix through a series of calculation, and the detailed process to construct the Darboux matrix can be found consulting [41].

In fact, the DT is a special gauge transformation, we can start from a trivial solution and get the nontrivial exact solutions by this method eventually. The basic ideas of DT is to use the seed solutions and the Lax pair, we get the DT with the aid of the gauge transformation in combination with the spectral problem. To be specific, the gauge transformation is

$$
\Psi^{[1]}=T_{1} \Psi,
$$

where the matrix $T$ can transform the Lax pair (6a)-(6b) into a new one, possessing the same form

$$
\Psi_{x}^{[1]}=U^{[1]} \Psi, \quad \Psi_{t}^{[1]}=V^{[1]} \Psi,
$$

where $U^{[1]}$ and $V^{[1]}$ have the same form as $U$ and $V$ but replacing $q, p$ with $q_{1}, p_{1}$. And $U^{[1]}, V^{[1]}$ satisfy the two equations

$$
T_{1 x}+T_{1} U=U^{[1]} T_{1}, \quad T_{1 t}+T_{1} V=V^{[1]} T_{1} .
$$

From equation (10), we can construct a basic DT matrix, and it is possible to find the relationship between the new potential function $q_{1}$ and the initial potential $q$. Referring to the method to get Darboux matrix $T$ of literature [41], using equation (10) and comparing the coefficients of $\lambda^{j}$, we can get the expression of $a_{0}, d_{0}$ in equation (7) as

$$
\begin{aligned}
& q_{1}=\frac{a_{1} q}{d_{1}}-\frac{2 i \beta b_{0}}{d_{1}\left(-\beta+\frac{(\alpha \beta+\sqrt{2} \omega) \beta}{2+\alpha \beta}\right)}, \quad p_{1}=\frac{d_{1} p}{a_{1}}+\frac{2 i c_{0} \beta}{a_{1}\left(-\beta-\frac{\sqrt{2} \omega \beta}{2}\right)}, \\
& a_{0}=\frac{-i(2 \sqrt{2}+4 \sqrt{-\alpha \beta}-\sqrt{2} \alpha \beta) a_{1}}{2 \beta(2+\sqrt{2} \omega)}, \quad d_{0}=\frac{-i(-2 \sqrt{2}+4 \sqrt{-\alpha \beta}+\sqrt{2} \alpha \beta) a_{1}}{2 \beta(-2+\sqrt{2} \omega)},
\end{aligned}
$$

moreover, $b_{0}, c_{0}$ are satisfied with

$$
\begin{array}{ll}
b_{0 x}=\frac{8 i b_{0}}{\beta(2+\sqrt{2} \omega)(-2+\sqrt{2} \omega)^{2}}, & b_{0 t}=-\frac{16 i b_{0} r}{(-2+\sqrt{2} \omega)^{3}(2+\sqrt{2} \omega)}, \\
c_{0 x}=\frac{8 i c_{0}}{\beta(2+\sqrt{2} \omega)(-2+\sqrt{2} \omega)^{2}}, & c_{0 t}=\frac{16 r i c_{0}}{(-2+\sqrt{2} \omega)(2+\sqrt{2} \omega)^{3}} .
\end{array}
$$

Solving equations (11) and (12), we have

$$
b_{0}=C_{11} e^{m_{9}}, \quad c_{0}=C_{12} e^{m_{10}},
$$

where

$$
\begin{aligned}
& C_{11}=\lambda_{1}-\frac{i(2 \sqrt{2}+4 \omega-\sqrt{2} \alpha \beta)}{2 \beta(2+\sqrt{2} \omega)}, \\
& C_{12}=\lambda_{1}-\frac{i(-2 \sqrt{2}+4 \omega+\sqrt{2} \alpha \beta)}{2 \beta(-2+\sqrt{2} \omega)},
\end{aligned}
$$




$$
\begin{aligned}
& m_{9}=\frac{-4 i(-2 r t \beta+\sqrt{2} r \omega \beta t+x \alpha \beta+2 \sqrt{2} \omega x-2 x)}{(\alpha \beta+2 \sqrt{2} \omega-2)(2+\alpha \beta)(-2+\sqrt{2} \omega) \beta}, \\
& m_{10}=\frac{-4 i(2 r t \beta+\sqrt{2} r \omega \beta t-x \alpha \beta+2 \sqrt{2} \omega x+2 x)}{(-\alpha \beta+2 \sqrt{2} \omega+2)(2+\alpha \beta)(2+\sqrt{2} \omega) \beta} .
\end{aligned}
$$

Now, we have gotten four parameters $a_{0}, b_{0}, c_{0}, d_{0}$, and the next work is to calculate the remaining two variables $a_{1}, d_{1}$. Then from the equation $\Psi^{[1]}=T_{1} \Psi$, there must be one $\lambda=\lambda_{1}$ satisfying $\left.T_{1}\right|_{\lambda=\lambda_{1}} \Psi_{1}=0$ [37], we get

$$
\left(a_{1} \lambda+a_{0}\right) \phi_{1}+b_{0} \varphi_{1}=0, \quad c_{0} \phi_{1}+\left(d_{1} \lambda+d_{0}\right) \varphi_{1}=0
$$

then $a_{1}=-\frac{\varphi_{1}}{\phi_{1}} e^{m 9}, d_{1}=-\frac{\phi_{1}}{\varphi_{1}} e^{m_{10}}$, now the Darboux matrix $T$ and the potential functions $q_{1}$, $p_{1}$ can be obtained explicitly,

$$
\begin{aligned}
& q_{1}=\frac{\varphi_{1}^{2}}{\phi_{1}^{2}} e^{\left(m_{9}-m_{10}\right)} q+2 i \frac{C_{11}}{\left(-\beta+\frac{(\alpha \beta+\sqrt{2} \sqrt{-\alpha \beta}) \beta}{2+\alpha \beta}\right)}, \\
& p_{1}=\frac{\phi_{1}^{2}}{\varphi_{1}^{2}} e^{\left(m_{10}-m_{9}\right)} p-2 i \frac{C_{12}}{\left(-\beta+\frac{\sqrt{2} \sqrt{-\alpha \beta} \beta}{2}\right)} .
\end{aligned}
$$

Now, we assume a $\tilde{\lambda}$, and in order to get a unified $\tilde{\lambda}$, we assume

$$
\frac{C_{11}}{\left(-\beta+\frac{(\alpha \beta+\sqrt{2} \sqrt{-\alpha \beta}) \beta}{2+\alpha \beta}\right)}-\frac{C_{12}}{\left(-\beta+\frac{\sqrt{2} \sqrt{-\alpha \beta} \beta}{2}\right)}=0,
$$

and we get

$$
\alpha=0,-\frac{\left(-\frac{3 \sqrt{2}}{2}-i \lambda \beta+\frac{\sqrt{-14-4 i \sqrt{2} \lambda \beta-4 \lambda^{2} \beta^{2}}}{2}\right)^{2}}{\beta},-\frac{\left(-\frac{3 \sqrt{2}}{2}-i \lambda \beta-\frac{\sqrt{-14-4 i \sqrt{2} \lambda \beta-4 \lambda^{2} \beta^{2}}}{2}\right)^{2}}{\beta} .
$$

In this section, we reduce the number of parameters and want one relatively simple $T$, without loss of generalization we can assume $\alpha=0$, and finally we can get

$$
\left.T_{1}\right|_{\lambda=\lambda_{1}}=\left(\begin{array}{cc}
-\frac{\varphi_{1}}{\phi_{1}} e^{\left(i\left(\frac{1}{\beta} x-\frac{1}{\beta^{2}} t\right)\right)}\left(\lambda-\frac{i \sqrt{2}}{2 \beta}\right) & \left(-\frac{i \sqrt{2}}{2 \beta}+\lambda_{1}\right) e^{\left(i\left(\frac{1}{\beta} x-\frac{1}{\beta^{2}} t\right)\right)} \\
\left(-\frac{i \sqrt{2}}{2 \beta}+\lambda_{1}\right) e^{\left(-i\left(\frac{1}{\beta} x-\frac{1}{\beta^{2}} t\right)\right)} & -\frac{\phi_{1}}{\varphi_{1}}\left(\lambda-\frac{i \sqrt{2}}{2 \beta}\right) e^{\left(-i\left(\frac{1}{\beta} x-\frac{1}{\beta^{2}} t\right)\right)}
\end{array}\right),
$$

and the relationships between the potential functions are as follows:

$$
\begin{aligned}
& q_{1}=q\left(\frac{\varphi_{1}}{\phi_{1}}\right)^{2} e^{\left(2 i\left(\frac{1}{\beta} x-\frac{1}{\beta^{2}} t\right)\right)}-2 i \frac{\varphi_{1}}{\phi_{1}}\left(-\frac{i \sqrt{2}}{2 \beta}+\lambda_{1}\right) e^{\left(2 i\left(\frac{1}{\beta} x-\frac{1}{\beta^{2}} t\right)\right)}, \\
& p_{1}=p\left(\frac{\phi_{1}}{\varphi_{1}}\right)^{2} e^{\left(-2 i\left(\frac{1}{\beta} x-\frac{1}{\beta^{2}} t\right)\right)}+2 i \frac{\phi_{1}}{\varphi_{1}}\left(-\frac{i \sqrt{2}}{2 \beta}+\lambda_{1}\right) e^{\left(-2 i\left(\frac{1}{\beta} x-\frac{1}{\beta^{2}} t\right)\right)},
\end{aligned}
$$

under the reduction conditions of $q^{*}=p$ and $\lambda_{1}^{*}=-\lambda_{1}$, substituting this relation into (13a)(13b), we have

$$
q_{1}^{*}=q^{*}\left(\frac{\phi_{1}}{\varphi_{1}}\right)^{2} e^{\left(-2 i\left(\frac{1}{\beta} x-\frac{1}{\beta^{2}} t\right)\right)}+2 i\left(\frac{\phi_{1}}{\varphi_{1}}\right)\left(-\frac{i \sqrt{2}}{2 \beta}+\lambda_{1}\right) e^{\left(-2 i\left(\frac{1}{\beta} x-\frac{1}{\beta^{2}} t\right)\right)}=p_{1},
$$


and the corresponding new eigenfunction

$$
\Psi_{j}^{[1]}=\left(\begin{array}{c}
\frac{1}{\phi_{1}}\left|\begin{array}{ll}
-\left(\lambda_{j}-\frac{i \sqrt{2}}{2 \beta}\right) \phi_{j} & \varphi_{j} \\
-\left(\lambda_{1}-\frac{i \sqrt{2}}{2 \beta}\right) \phi_{1} & \varphi_{1}
\end{array}\right| e^{\left(i\left(\frac{1}{\beta} x-\frac{1}{\beta^{2}} t\right)\right)} \\
\frac{1}{\varphi_{1}}\left|\begin{array}{ll}
-\left(\lambda_{j}-\frac{i \sqrt{2}}{2 \beta}\right) \varphi_{j} & \phi_{j} \\
-\left(\lambda_{j}-\frac{i \sqrt{2}}{2 \beta}\right) \varphi_{1} & \phi_{1}
\end{array}\right| e^{\left(-i\left(\frac{1}{\beta} x-\frac{1}{\beta^{2}} t\right)\right)}
\end{array}\right) .
$$

It is straightforward to verify that when $j=1$, the $\Psi_{j}^{[1]}=\left.T_{1}\right|_{\lambda=\lambda_{1}} \Psi_{j}=0$, this fact implies that $\lambda_{1}$ may not be used more than once when considering the iterations for the DT. But Matveev and Salle [2] have pointed out that a generalized DT does exist, we can use the method to solve the rational solution of equation (5).

In order to get the DT of more than two orders, we translate the spectral parameter $\tilde{\lambda}_{1}=-\frac{i \sqrt{2}}{2 \beta}+\lambda$ and $\tilde{\lambda}_{j}=-\frac{i \sqrt{2}}{2 \beta}+\lambda_{j}$. We assume

$$
\begin{aligned}
& n=1, \quad T_{1}=P_{1} \tilde{\lambda}+P_{0}=\left(\begin{array}{cc}
a_{1} \tilde{\lambda} & b_{0} \\
c_{0} & d_{1} \tilde{\lambda}
\end{array}\right), \\
& n=2, \quad T_{2}=P_{2} \tilde{\lambda}^{2}+P_{1} \tilde{\lambda}+P_{0}=\left(\begin{array}{cc}
a_{2} & 0 \\
0 & d_{2}
\end{array}\right) \tilde{\lambda}^{2}+\left(\begin{array}{cc}
0 & b_{1} \\
c_{1} & 0
\end{array}\right) \tilde{\lambda}+\left(\begin{array}{cc}
a_{0} & 0 \\
0 & d_{0}
\end{array}\right),
\end{aligned}
$$

similarly $T_{n}=\sum_{l=0}^{n} P_{l} \tilde{\lambda}^{l}$, where

$$
\begin{aligned}
& P_{n}=\left(\begin{array}{cc}
a_{n} & 0 \\
0 & d_{n}
\end{array}\right) \in\left\{\left(\begin{array}{ll}
a & 0 \\
0 & d
\end{array}\right)\right\}, \\
& P_{n-1}=\left(\begin{array}{cc}
0 & b_{n} \\
c_{n} & 0
\end{array}\right) \in\left\{\left(\begin{array}{ll}
0 & b \\
c & 0
\end{array}\right)\right\},
\end{aligned}
$$

$a, b, c, d$ are complex functions of $(x, t) . P_{0}$ is a constant matrix when $n$ is even and $P_{0}$ is a matrix that is known. Similar to one-fold DT, we can construct the $T_{n}$ and it is given in Appendix 1.

Based on the $n$-fold Darboux transformation in [49], we just consider the condition of $n$ being even, through the $n$-fold DT $T_{n}$, we get

$$
q^{[n]}=\frac{\Omega_{n 1}^{2}}{\Omega_{n 3}^{2}} q-2 i \frac{\Omega_{n 1} \Omega_{n 2}}{\Omega_{n 3}^{2}}, \quad p^{[n]}=\frac{\Omega_{n 3}^{2}}{\Omega_{n 1}^{2}} p+2 i \frac{\Omega_{n 3} \Omega_{n 4}}{\Omega_{n 1}^{2}},
$$

here, for $n=2 k$,

$$
\Omega_{n 1}=\left|\begin{array}{ccccc}
\tilde{\lambda}_{1}^{n-1} \varphi_{1} & \tilde{\lambda}_{1}^{n-2} \phi_{1} & \ldots & \tilde{\lambda}_{1} \varphi_{1} & \phi_{1} \\
\tilde{\lambda}_{2}^{n-1} \varphi_{2} & \tilde{\lambda}_{2}^{n-2} \phi_{2} & \cdots & \tilde{\lambda}_{2} \varphi_{2} & \phi_{2} \\
\vdots & \vdots & \vdots & \vdots & \vdots \\
\tilde{\lambda}_{n-1}^{n-1} \varphi_{n-1} & \tilde{\lambda}_{n-1}^{n-2} \phi_{n-1} & \cdots & \tilde{\lambda}_{n-1} \varphi_{n-1} & \phi_{n-1} \\
\tilde{\lambda}_{n}^{n-1} \varphi_{n} & \widetilde{\lambda}_{n}^{n-2} \phi_{n} & \cdots & \widetilde{\lambda}_{n} \varphi_{n} & \phi_{n}
\end{array}\right|,
$$




$$
\begin{aligned}
& \Omega_{n 2}=\left|\begin{array}{ccccc}
\tilde{\lambda}_{1}^{n} \phi_{1} & \tilde{\lambda}_{1}^{n-2} \phi_{1} & \ldots & \tilde{\lambda}_{1} \varphi_{1} & \phi_{1} \\
\tilde{\lambda}_{2}^{n} \phi_{2} & \tilde{\lambda}_{2}^{n-2} \phi_{2} & \ldots & \tilde{\lambda}_{2} \varphi_{2} & \phi_{2} \\
\vdots & \vdots & \vdots & \vdots & \vdots \\
\tilde{\lambda}_{n-1}^{n} \phi_{n-1} & \tilde{\lambda}_{n-1}^{n-2} \phi_{n-1} & \ldots & \tilde{\lambda}_{n-1} \varphi_{n-1} & \phi_{n-1} \\
\widetilde{\lambda}_{n}^{n} \phi_{n} & \widetilde{\lambda}_{n}^{n-2} \phi_{n} & \ldots & \widetilde{\lambda}_{n} \varphi_{n} & \phi_{n}
\end{array}\right|, \\
& \Omega_{n 3}=\left|\begin{array}{ccccc}
\tilde{\lambda}_{1}^{n-1} \phi_{1} & \tilde{\lambda}_{1}^{n-2} \varphi_{1} & \ldots & \tilde{\lambda}_{1} \phi_{1} & \varphi_{1} \\
\tilde{\lambda}_{2}^{n-1} \phi_{2} & \widetilde{\lambda}_{2}^{n-2} \varphi_{2} & \ldots & \tilde{\lambda}_{2} \phi_{2} & \varphi_{2} \\
\vdots & \vdots & \vdots & \vdots & \vdots \\
\tilde{\lambda}_{n-1}^{n-1} \phi_{n-1} & \tilde{\lambda}_{n-1}^{n-2} \varphi_{n-1} & \ldots & \tilde{\lambda}_{n-1} \phi_{n-1} & \varphi_{n-1} \\
\tilde{\lambda}_{n}^{n-1} \phi_{n} & \widetilde{\lambda}_{n}^{n-2} \varphi_{n} & \ldots & \tilde{\lambda}_{n} \phi_{n} & \varphi_{n}
\end{array}\right|, \\
& \Omega_{n 1}=\left|\begin{array}{ccccc}
\tilde{\lambda}_{1}^{n} \varphi_{1} & \tilde{\lambda}_{1}^{n-2} \varphi_{1} & \ldots & \tilde{\lambda}_{1} \phi_{1} & \varphi_{1} \\
\tilde{\lambda}_{2}^{n} \varphi_{2} & \tilde{\lambda}_{2}^{n-2} \varphi_{2} & \ldots & \tilde{\lambda}_{2} \phi_{2} & \varphi_{2} \\
\vdots & \vdots & \vdots & \vdots & \vdots \\
\tilde{\lambda}_{n-1}^{n} \varphi_{n-1} & \tilde{\lambda}_{n-1}^{n-2} \varphi_{n-1} & \ldots & \tilde{\lambda}_{n-1} \phi_{n-1} & \varphi_{n-1} \\
\widetilde{\lambda}_{n}^{n} \phi_{n} & \widetilde{\lambda}_{n}^{n-2} \varphi_{n} & \ldots & \widetilde{\lambda}_{n} \phi_{n} & \varphi_{n}
\end{array}\right|,
\end{aligned}
$$

under this condition of $\lambda_{2}=-\lambda_{1}^{*}, \Psi_{2}=\left(\begin{array}{c}-\varphi_{1}^{*} \\ \phi_{1}^{*}\end{array}\right)$, the two-fold form is

$$
q^{[2]}=\frac{\Omega_{21}^{2}}{\Omega_{23}^{2}} q-2 i \frac{\Omega_{21} \Omega_{22}}{\Omega_{23}^{2}}
$$

at the same time $\Psi_{2}$ can also satisfy equations (6a)-(6b). Substituting $\Psi_{1}, \Psi_{2}$ into (15), we get

$$
\begin{aligned}
& \Omega_{21}=\tilde{\lambda}_{1} \varphi_{1} \phi_{2}-\tilde{\lambda}_{2} \phi_{1} \varphi_{2}=-\tilde{\lambda}_{1} \varphi_{1} \varphi_{1}^{*}+\tilde{\lambda}_{1}^{*} \phi_{1} \phi_{1}^{*}, \\
& \Omega_{22}=\left(\tilde{\lambda}_{1}^{2}-\tilde{\lambda}_{2}^{2}\right) \phi_{1} \phi_{2}=-\left(\widetilde{\lambda}_{1}^{2}-\tilde{\lambda}_{1}^{* 2}\right) \phi_{1} \varphi_{1}^{*}, \\
& \Omega_{23}=\tilde{\lambda}_{1} \phi_{1} \varphi_{2}-\tilde{\lambda}_{2} \varphi_{1} \phi_{2}=\tilde{\lambda}_{1} \phi_{1} \phi_{1}^{*}-\tilde{\lambda}_{1}^{*} \varphi_{1} \varphi_{1}^{*},
\end{aligned}
$$

then equation (14) can be written as

$$
q^{[2]}=\frac{\left(-\tilde{\lambda}_{1}\left|\varphi_{1}\right|^{2}+\widetilde{\lambda}_{1}^{*}\left|\phi_{1}\right|^{2}\right)^{2}}{\left(\widetilde{\lambda}_{1}\left|\phi_{1}\right|^{2}-\widetilde{\lambda}_{1}^{*}\left|\varphi_{1}\right|^{2}\right)^{2}} q-2 i \frac{-\left(\widetilde{\lambda}_{1}^{2}-\widetilde{\lambda}_{1}^{* 2}\right)\left(-\tilde{\lambda}_{1}\left|\varphi_{1}\right|^{2}+\tilde{\lambda}_{1}^{*}\left|\phi_{1}\right|^{2}\right) \phi_{1} \varphi_{1}^{*}}{\left(\widetilde{\lambda}_{1}\left|\phi_{1}\right|^{2}-\widetilde{\lambda}_{1}^{*}\left|\varphi_{1}\right|^{2}\right)^{2}}
$$

Equations (14) and (16) give us the new solutions after a Darboux transformation, and we can use the results of (14) and (15) to get two-order and three-order rogue wave solutions. In the next section, we will consider the condition of the degeneration case of the Darboux matrix $T_{2 k}$ to get the exact rogue wave solutions of equation (5). In the case of degeneration, the $q^{[2 k]}$ can be expressed as an infinite expression which is of the form of $\frac{0}{0}$, then we can make a Taylor expansion at $\tilde{\lambda}=\tilde{\lambda}_{0}$ and get the smooth solutions of equation (5).

\section{Solutions of rogue wave}

In order to get the rational solution, we can apply the generalized Darboux transformation. We start with the seed solution of equation (5),

$$
q=c_{1} e^{i\left(a_{1} x-\left(\alpha a_{1}-a_{1}^{2}+c_{1}^{2}-a_{1} c_{1}^{2} \beta+r\right) t\right)} .
$$


Substituting (17) into the spectral equations (6a)-(6b), we have the corresponding solution for the spectral problem,

$$
\Psi_{1}(f)=\left(\begin{array}{c}
\left(C_{2} \mathrm{e}^{-A}+C_{1} \mathrm{e}^{A}\right) \mathrm{e}^{\frac{i}{2}\left(a_{1} x-\left(\alpha a_{1}-a_{1}{ }^{2}+c_{1}{ }^{2}-\beta c_{1}{ }^{2} a_{1}+r\right) t\right)} \\
-2(2+\alpha \beta)\left(\frac{C_{2} \mathrm{e}^{-A}\left(-\frac{i}{16} A_{1}+M_{2}\right)}{M_{1}}+\frac{C_{1} \mathrm{e}^{A}\left(\frac{i}{16} A_{1}+M_{2}\right)}{M_{1}}\right) \mathrm{e}^{-\frac{i}{2}\left(a_{1} x-\left(\alpha a_{1}-a_{1}{ }^{2}+c_{1}{ }^{2}-\beta c_{1}{ }^{2} a_{1}+r\right) t\right)}
\end{array}\right),
$$

where

$$
\begin{aligned}
A= & \frac{A_{1}\left(x+\frac{i t}{8}\left(16 \sqrt{2} \lambda+8 i c_{1}^{2} \beta+8 i a_{1}+4 i \alpha+16 i \lambda^{2} \beta\right)\right)}{16 \sqrt{2} \lambda+8 i c_{1}^{2} \beta+8 i a_{1}+4 i \alpha+16 i \lambda^{2} \beta}, \\
A_{1}= & 4\left(\left(16 \lambda c_{1}^{2} \beta+16 \lambda a_{1}+8 \lambda \alpha\right)(\sqrt{2} i-\lambda \beta)-32 \sqrt{2} i \lambda^{3} \beta-8 \lambda^{2} \alpha \beta-32 \lambda^{2}+16 \lambda^{4} \beta^{2}\right. \\
& \left.+4 a_{1}^{2}+8 c_{1}^{2}+\alpha^{2}+4 a_{1} \alpha+4 c_{1}^{2} \alpha \beta\right)^{\frac{1}{2}}\left(-2 a_{1}-2 c_{1}^{2} \beta-4 \lambda^{2} \beta+4 \sqrt{2} i \lambda-\alpha\right), \\
M_{1}= & \frac{1}{4}\left(8 \sqrt{2} \omega \beta^{2} \lambda^{3}+4 \sqrt{2} \omega \beta^{2} c_{1}^{2}+4 \sqrt{2} \omega \lambda a_{1} \beta\right. \\
& +2 \sqrt{2} \omega \lambda \alpha \beta+2 \sqrt{2} i \alpha+4 \sqrt{2} i a_{1}+2 \sqrt{2} i c_{1}^{2} \alpha \beta^{2} \\
& -16 i \lambda^{2} \omega \beta+24 \sqrt{2} i \lambda^{2} \beta+2 \sqrt{2} i a_{1} \alpha \beta+4 \sqrt{2} i \lambda^{2} \alpha \beta^{2}+4 \sqrt{2} i c_{1}^{2} \beta \\
& \left.+\sqrt{2} i \alpha^{2} \beta+16 \lambda-8 \lambda c_{1}^{2} \beta^{2}-16 \beta^{2} \lambda^{3}+4 a \lambda \beta-8 a_{1} \beta \lambda\right) c_{1}, \\
M_{2}= & \frac{\sqrt{2}}{2} \lambda \beta c_{1}^{2}+2 \sqrt{2} \lambda^{3} \beta+\frac{i}{2} c_{1}^{2} \lambda^{2} \beta^{2}+i \lambda^{4} \beta^{2}-\frac{i}{4} c_{1}^{2} a_{1} \beta-\frac{i}{8} c_{1}^{2} \alpha \beta \\
& -\frac{i}{4} a_{1} \alpha-\frac{i}{16} \alpha^{2}-\frac{1}{4} i a_{1}^{2}-2 i \lambda^{2} .
\end{aligned}
$$

Next we will simplify the result; we assume $r=1, a_{1}=0, c_{1}=1, \beta=1, \alpha=0$, while $q=e^{2 i t}$, and $\lambda=\frac{1}{2}+\frac{i}{2}+\frac{\sqrt{2} i}{2}$ is only one zero point of the $A_{1}, f$ limit to 0 , and assuming $\lambda=\frac{1}{2}+i h$, now

$$
\begin{aligned}
& A_{1}=\sqrt{64 i \lambda\left(\lambda^{6}-2 \lambda^{4}-\frac{1}{8} \lambda^{2}+\frac{1}{16}\right) \sqrt{2}+2+16 \lambda^{8}-192 \lambda^{6}+60 \lambda^{4}+12 \lambda^{2}}, \\
& A=\frac{A_{1}\left(x+i\left(2 \sqrt{2} \lambda+2 i \lambda^{2}+i\right) t\right)}{4 \sqrt{2} \lambda+4 i \lambda^{2}+2 i}, \\
& C_{1}=-\frac{h_{1}+\sqrt{h_{1}^{2}-1}}{\sqrt{h_{1}^{2}-1}}, \quad C_{2}=\frac{h_{1}-\sqrt{h_{1}^{2}-1}}{\sqrt{h_{1}^{2}-1}}, \\
& h=\frac{1}{2}+\frac{\sqrt{2}}{2}+f^{2}, \quad h_{1}=2 \sqrt{2} \lambda+2 i \lambda^{2}+i .
\end{aligned}
$$

We Taylor expand the vector function $\Psi_{1}(f)$ at $f=0$,

$$
\Psi_{1}(f)=\Psi_{1}^{[0]}+\Psi_{1}^{[1]} f^{2}+\Psi_{1}^{[2]} f^{3}+\cdots+\Psi_{1}^{[N]} f^{N}+\cdots
$$

where

$$
\Psi_{1}^{[0]}=\left(\begin{array}{c}
\left(\frac{1}{2}-\frac{1}{2} i\right) \sqrt{-1-i} e^{i t} \\
\left(\frac{1}{2}+\frac{1}{2} i\right) \sqrt{-1-i} e^{-i t}
\end{array}\right),
$$




$$
\Psi_{1}^{[1]}=\left(\begin{array}{c}
\left(-\frac{1}{4}+\frac{1}{4} i\right) \sqrt{1-7 i}(-x+2 t+i t) e^{i t} \\
\left(-\frac{1}{20}-\frac{1}{10} i\right) \sqrt{1-7 i}(7 t-3 x-4-2 i+i t+i x) e^{-i t}
\end{array}\right) .
$$

Substituting $\beta=1, \Psi_{1}^{[0]}$ into (13a)-(13b), we get $q_{1}=i e^{(-2 i(-x+2 t))}$ and $p_{1}=-i e^{(2 i(-x+2 t))}$, while $q_{1}$ and $p_{1}$ are not rogue wave solutions that we prefer, so we consider two-fold DT to get the new solution $q^{[2]}$.

Assume $a_{1}=\left.\frac{\partial \varphi_{1}\left(\lambda_{0}+f\right)}{\partial f}\right|_{f=0}, b_{1}=\left.\frac{\partial \phi_{1}\left(\lambda_{0}+f\right)}{\partial f}\right|_{f=0}$ by a Taylor expansion, we plug $\Psi_{1}^{[1]}$ into equation (16),

$$
q_{\mathrm{rw}}^{[1]}=\frac{\left(-\tilde{\lambda}_{0}\left|a_{1}\right|^{2}+\tilde{\lambda}_{0}^{*}\left|b_{1}\right|^{2}\right)^{2}}{\left(\widetilde{\lambda}_{0}\left|b_{1}\right|^{2}-\widetilde{\lambda}_{0}^{*}\left|a_{1}\right|^{2}\right)^{2}} q-2 i \frac{-\left(\tilde{\lambda}_{0}^{2}-\widetilde{\lambda}_{0}^{* 2}\right)\left(-\tilde{\lambda}_{0}\left|a_{1}\right|^{2}+\tilde{\lambda}_{0}^{*}\left|b_{1}\right|^{2}\right) \phi_{1} \varphi_{1}^{*}}{\left(\widetilde{\lambda}_{0}\left|b_{1}\right|^{2}-\widetilde{\lambda}_{0}^{*}\left|a_{1}\right|^{2}\right)^{2}}
$$

then we obtain the first-order rational solution $q_{\text {rational }}^{[1]}$, which means the one-order rogue wave solution is

$$
q_{\mathrm{rw}}^{[1]}=\frac{G_{1} G_{2}}{H^{2}} e^{2 i t},
$$

with

$$
\begin{aligned}
& G_{1}=-\left(-3 t+1+i+x+i x^{2}-4 i x t+i x+5 i t^{2}-3 i t\right), \\
& G_{2}=\left(5 i t^{2}-3 i t-1-i-4 i x t+i x+i x^{2}+t+x\right), \\
& H=\left(3 t-1+i-x+i x^{2}-4 i x t+i x+5 i t^{2}-3 i t\right) .
\end{aligned}
$$

The plot of this solution is shown in Figures 1 and 2 . Figure 1 is the three-dimensional plot of $\left|q_{\mathrm{rw}}^{[1]}\right|$ with $r=1, a_{1}=0, c_{1}=1, \beta=1, \alpha=0$, and Figure 2 is the corresponding density plot. It is shown that the first-order rogue wave solution is localized in both space and time.

Next we calculate the $q_{\mathrm{rw}}^{[2]}$, through the above procedure,

$$
\begin{aligned}
q_{\mathrm{rw}}^{[2]}= & \frac{\left(-\widetilde{\lambda}_{0}\left|a_{2}^{[2]}\right|^{2}+\widetilde{\lambda}_{0}^{*}\left|b_{2}^{[2]}\right|^{2}\right)^{2}}{\left(\widetilde{\lambda}_{0}\left|b_{2}^{[2]}\right|^{2}-\widetilde{\lambda}_{0}^{*}\left|a_{2}^{[2]}\right|^{2}\right)^{2}} q^{[2]} \\
& -2 i \frac{\left(\widetilde{\lambda}_{0}^{2}-\widetilde{\lambda}_{0}^{* 2}\right)\left(-\widetilde{\lambda}_{0}\left|a_{2}^{[2]}\right|^{2}+\widetilde{\lambda}_{0}^{*}\left|b_{2}^{[2]}\right|^{2}\right) b_{2}^{[2]}\left(a_{2}^{[2]}\right)^{*}}{\left(\widetilde{\lambda}_{0}\left|b_{2}^{[2]}\right|^{2}-\widetilde{\lambda}_{0}^{*}\left|a_{2}^{[2]}\right|^{2}\right)^{2}}
\end{aligned}
$$

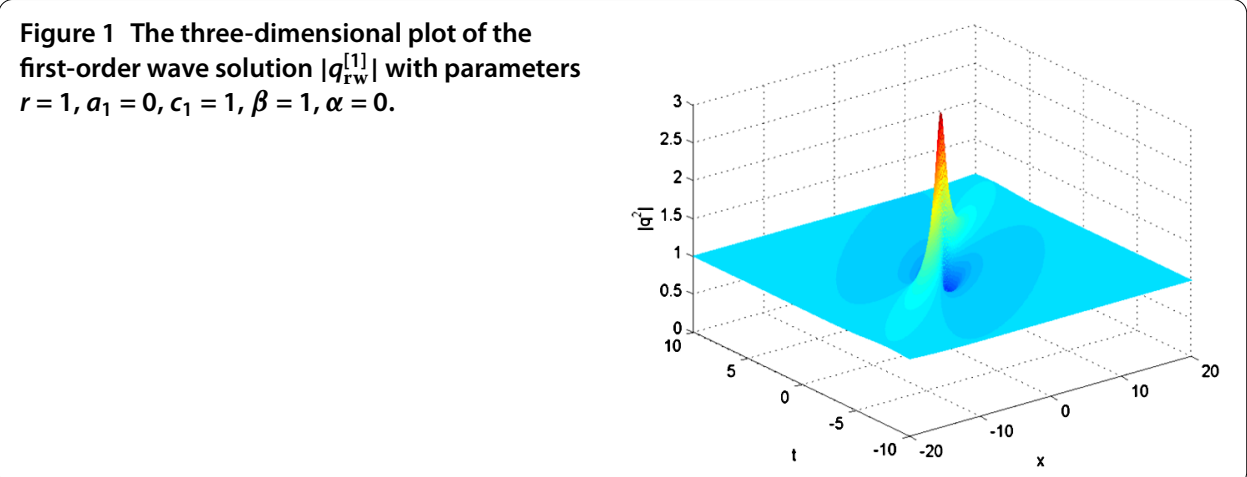




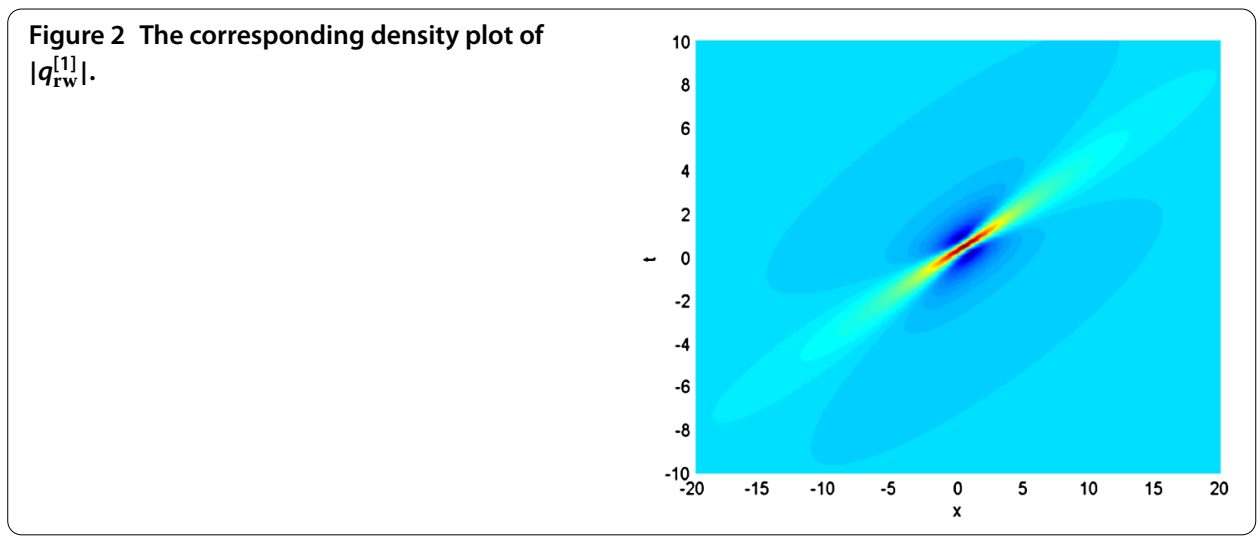

Figure 3 The three-dimensional plot of the two-order wave solution $\left|q_{\mathrm{rw}}^{[2]}\right|$ with parameters $r=1, a_{1}=0, c_{1}=1, \beta=1, \alpha=0$.

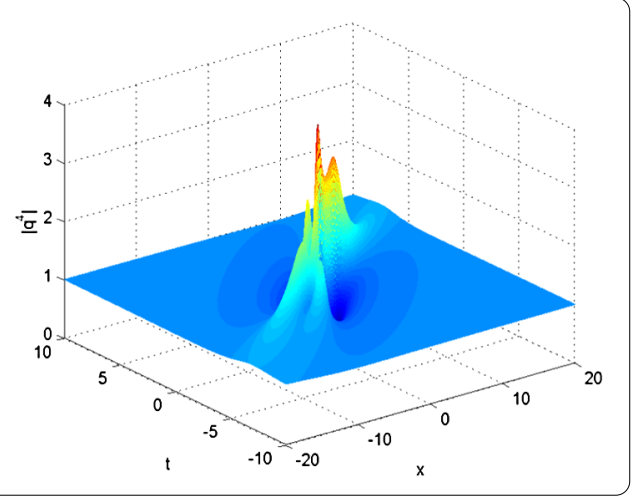

with

$$
\begin{gathered}
b_{2}^{[2]}=\frac{\left|\begin{array}{ccc}
\frac{\partial^{2}}{\partial f^{2}}\left(\left(\widetilde{\lambda}_{0}+i f^{2}\right)^{2} \phi_{1}\left(\lambda_{0}+f\right)\right) & \frac{\partial^{2}}{\partial f^{2}}\left(\left(\widetilde{\lambda}_{0}+i f^{2}\right) \varphi_{1}\left(\lambda_{0}+f\right)\right) & \frac{\partial^{2}}{\partial f^{2}} \phi_{1}\left(\lambda_{0}+f\right) \\
\widetilde{\lambda}_{0}^{2} b_{1} & \widetilde{\lambda}_{0} a_{1} & b_{1} \\
-\left(\widetilde{\lambda}_{0}^{2} a_{1}\right)^{*} & -\left(\widetilde{\lambda}_{0} b_{1}\right)^{*} & -a_{1}^{*}
\end{array}\right|}{\left|\begin{array}{ccc}
\widetilde{\lambda}_{0} b_{1} & a_{1} \\
\left(\widetilde{\lambda}_{0} a_{1}\right)^{*} & b_{1}^{*}
\end{array}\right|} \\
a_{2}^{[2]}=\frac{\left|\begin{array}{ccc}
\left.\frac{\partial^{2}}{\partial f^{2}}\left(\left(\widetilde{\lambda}_{0}+i f^{2}\right)^{2} \varphi_{1}\left(\lambda_{0}+f\right)\right)\right) & \frac{\partial^{2}}{\partial f^{2}}\left(\left(\widetilde{\lambda}_{0}+i f^{2}\right) \phi_{1}\left(\lambda_{0}+f\right)\right) & \frac{\partial^{2}}{\partial f^{2}} \varphi_{1}\left(\lambda_{0}+f\right) \\
\widetilde{\lambda}_{0}^{2} a_{1} & \widetilde{\lambda}_{0} b_{1} & a_{1} \\
\left(\widetilde{\lambda}_{0}^{2} b_{1}\right)^{*} & \left(\widetilde{\lambda}_{0} a_{1}\right)^{*} & b_{1}^{*}
\end{array}\right|}{\left|\begin{array}{cc}
\tilde{\lambda}_{0} a_{1} & b_{1} \\
-\left(\widetilde{\lambda}_{0} b_{1}\right)^{*} & -a_{1}^{*}
\end{array}\right|}
\end{gathered}
$$

Substituting $a_{2}^{[2]}, b_{2}^{[2]}$ into equation (19), we get the two-order rogue wave solution

$$
q_{\mathrm{rw}}^{[2]}=\frac{-G_{1} G_{3} G_{4}}{H^{2} H_{1}^{2}} e^{2 i t},
$$

$G_{3}, G_{4}, H_{1}$ are given in Appendix 2. The plot of this solution is shown in Figures 3 and 4 . Figure 3 is the three-dimensional plot of $\left|q_{\mathrm{rw}}^{[2]}\right|$ with $r=1, a_{1}=0, c_{1}=1, \beta=1, \alpha=0$, and Figure 4 is the corresponding density plot. 


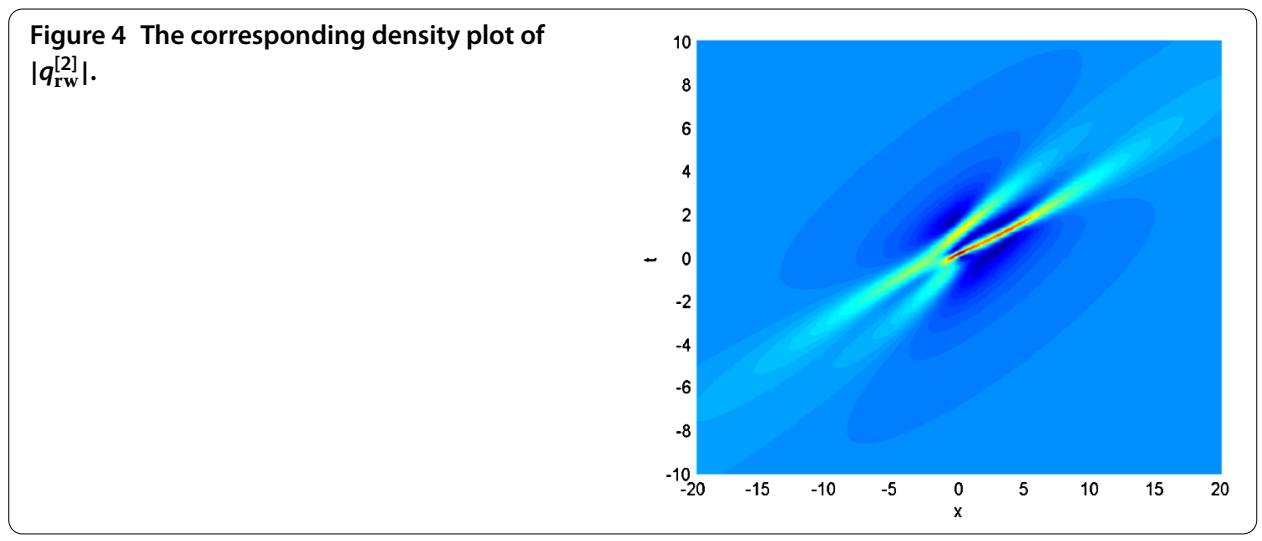

In the same way, the three-order solution can be given as

$$
q_{\mathrm{rw}}^{[3]}=\frac{\left(-\widetilde{\lambda}_{0}\left|a_{3}^{[4]}\right|^{2}+\widetilde{\lambda}_{0}^{*}\left|b_{3}^{[4]}\right|^{2}\right)^{2}}{\left(\widetilde{\lambda}_{0}\left|b_{3}^{[4]}\right|^{2}-\widetilde{\lambda}_{0}^{*}\left|a_{3}^{[4]}\right|^{2}\right)^{2}} q^{[4]}-2 i \frac{\left(\widetilde{\lambda}_{0}^{2}-\widetilde{\lambda}_{0}^{* 2}\right)\left(-\widetilde{\lambda}_{0}\left|a_{3}^{[4]}\right|^{2}+\widetilde{\lambda}_{0}^{*}\left|b_{3}^{[4]}\right|^{2}\right) b_{3}^{[4]}\left(a_{3}^{[4]}\right)^{*}}{\left(\widetilde{\lambda}_{0}\left|b_{3}^{[4]}\right|^{2}-\widetilde{\lambda}_{0}^{*}\left|a_{3}^{[4]}\right|^{2}\right)^{2}}
$$

Through the Darboux transformation, the eigenfunction $\psi_{j}^{[n]}=T_{n} \psi_{j}$ can be given. We can give the $\psi_{j}^{[4]}$ as follows:

$$
\psi_{j}^{[4]}=\frac{\frac{R M_{1}}{\Omega_{43}}}{\frac{R M_{2}}{\Omega_{41}}},
$$

where

$$
\begin{aligned}
& R M_{1}=\left|\begin{array}{ccccc}
\tilde{\lambda}_{j}^{4} \phi_{j} & \tilde{\lambda}_{j}^{3} \varphi_{j} & \tilde{\lambda}_{j}^{2} \phi_{j} & \tilde{\lambda}_{j} \varphi_{j} & \phi_{j} \\
\tilde{\lambda}_{1}^{4} \phi_{1} & \tilde{\lambda}_{1}^{3} \varphi_{1} & \tilde{\lambda}_{1}^{2} \phi_{1} & \tilde{\lambda}_{1} \varphi_{1} & \phi_{1} \\
\tilde{\lambda}_{2}^{4} \phi_{2} & \widetilde{\lambda}_{2}^{3} \varphi_{2} & \widetilde{\lambda}_{2}^{2} \phi_{2} & \tilde{\lambda}_{2} \varphi_{2} & \phi_{2} \\
\tilde{\lambda}_{3}^{4} \phi_{3} & \widetilde{\lambda}_{3}^{3} \varphi_{3} & \widetilde{\lambda}_{3}^{2} \phi_{3} & \tilde{\lambda}_{3} \varphi_{3} & \phi_{3} \\
\widetilde{\lambda}_{4}^{4} \phi_{4} & \widetilde{\lambda}_{4}^{3} \varphi_{4} & \widetilde{\lambda}_{4}^{2} \phi_{4} & \tilde{\lambda}_{4} \varphi_{4} & \phi_{4}
\end{array}\right|, \\
& R M_{2}=\left|\begin{array}{ccccc}
\widetilde{\lambda}_{j}^{4} \varphi_{j} & \widetilde{\lambda}_{j}^{3} \phi_{j} & \widetilde{\lambda}_{j}^{2} \varphi_{j} & \tilde{\lambda}_{j} \phi_{j} & \varphi_{j} \\
\tilde{\lambda}_{1}^{4} \varphi_{1} & \widetilde{\lambda}_{1}^{3} \phi_{1} & \widetilde{\lambda}_{1}^{2} \varphi_{1} & \tilde{\lambda}_{1} \phi_{1} & \varphi_{1} \\
\tilde{\lambda}_{2}^{4} \varphi_{2} & \widetilde{\lambda}_{2}^{3} \phi_{2} & \widetilde{\lambda}_{2}^{2} \varphi_{2} & \tilde{\lambda}_{2} \phi_{2} & \varphi_{2} \\
\tilde{\lambda}_{3}^{4} \varphi_{3} & \widetilde{\lambda}_{3}^{3} \phi_{3} & \widetilde{\lambda}_{3}^{2} \varphi_{3} & \widetilde{\lambda}_{3} \phi_{3} & \varphi_{3} \\
\widetilde{\lambda}_{4}^{4} \varphi_{4} & \widetilde{\lambda}_{4}^{3} \phi_{4} & \widetilde{\lambda}_{4}^{2} \varphi_{4} & \widetilde{\lambda}_{4} \phi_{4} & \varphi_{4}
\end{array}\right|,
\end{aligned}
$$

and $a_{3}^{[4]}, b_{3}^{[4]}$ are given as follows:

$$
b_{3}^{[4]}=\frac{\left|\begin{array}{ccccc}
r m_{41}^{3} & r m_{32}^{3} & r m_{21}^{3} & r m_{12}^{3} & r m_{01}^{3} \\
r m_{41}^{1} & r m_{32}^{1} & r m_{21}^{1} & r m_{12}^{1} & r m_{01}^{1} \\
-r m_{41}^{1 *} & -r m_{32}^{1 *} & -r m_{21}^{1 *} & -r m_{12}^{1 *} & -r m_{01}^{1 *} \\
r m_{41}^{2} & r m_{32}^{2} & r m_{21}^{2} & r m_{12}^{2} & r m_{01}^{2} \\
-r m_{32}^{2 *} & -r m_{21}^{2 *} & -r m_{12}^{2 *} & -r m_{01}^{2 *}
\end{array}\right|}{\left|\begin{array}{cccc}
r m_{31}^{1} & r m_{22}^{1} & r m_{11}^{1} & r m_{02}^{1} \\
-r m_{31}^{1 *} & -r m_{22}^{1 *} & -r m_{11}^{1 *} & -r m_{02}^{1 *} \\
r m_{31}^{2} & r m_{22}^{2} & r m_{11}^{2} & r m_{02}^{2} \\
-r m_{31}^{2 *} & -r m_{22}^{2 *} & -r m_{11}^{2 *} & -r m_{02}^{2 *}
\end{array}\right|}
$$



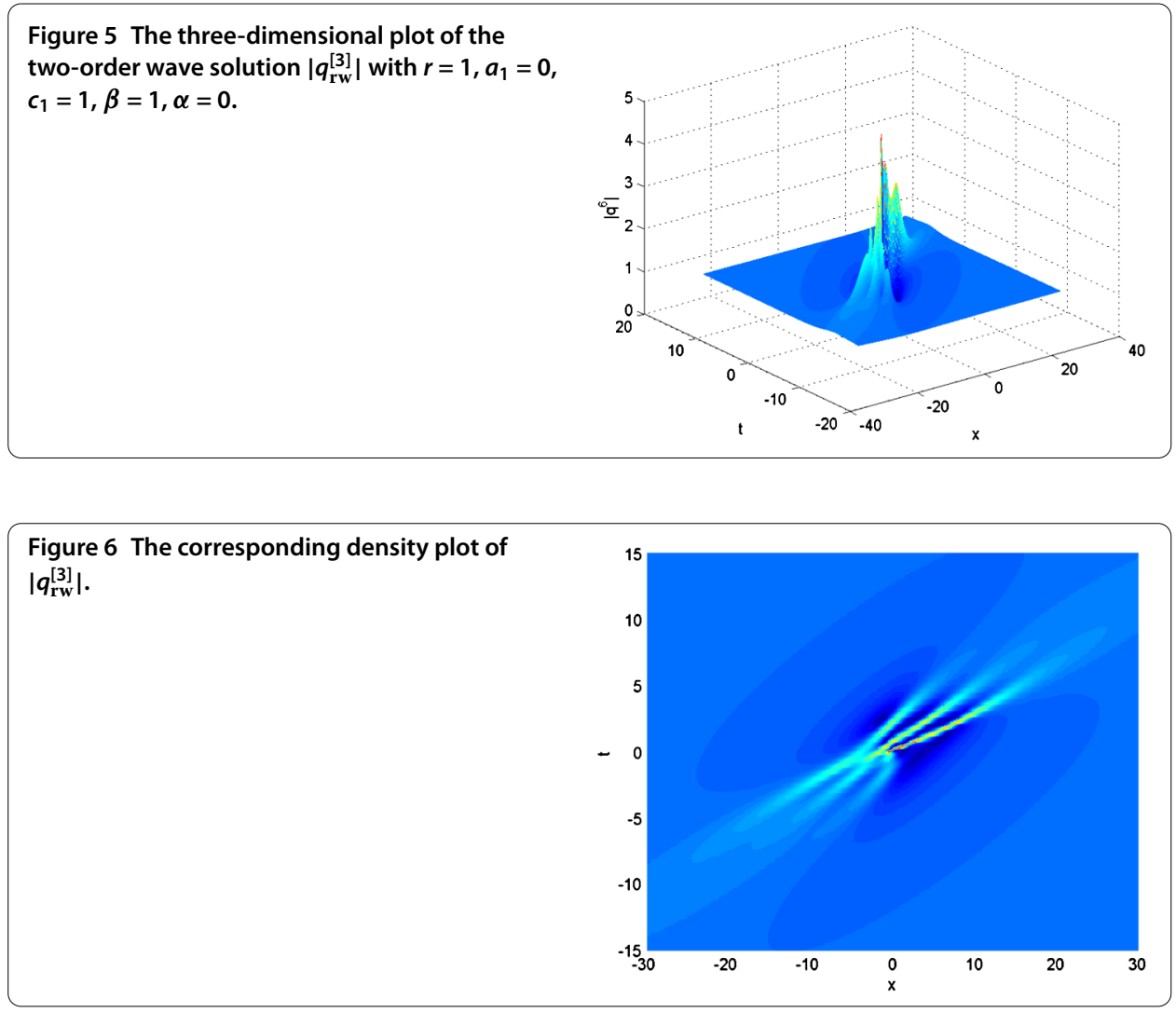

$$
a_{3}^{[4]}=\frac{\left|\begin{array}{ccccc}
r m_{42}^{3} & r m m_{31}^{3} & r m_{22}^{3} & r m_{11}^{3} & r m_{02}^{3} \\
r m_{42}^{1} & r m_{31}^{1} & r m_{22}^{1} & r m_{11}^{1} & r m_{02}^{1} \\
-r m_{42}^{1 *} & -r m_{31}^{1 *} & -r m_{22}^{1 *} & -r m_{11}^{1 *} & -r m_{02}^{1 *} \\
r m_{42}^{2} & r m_{31}^{2} & r m_{22}^{2} & r m_{11}^{2} & r m_{02}^{2} \\
-r m_{42}^{2 *} & -r m_{31}^{2 *} & -r m_{22}^{2 *} & -r m_{11}^{2 *} & -r m_{02}^{2 *}
\end{array}\right|}{\left|\begin{array}{cccc}
r m_{32}^{1} & r m_{21}^{1} & r m_{12}^{1} & r m_{01}^{1} \\
-r m_{32}^{1 *} & -r m_{21}^{1 *} & -r m_{12}^{1 *} & -r m_{01}^{1 *} \\
r m_{32}^{2} & r m_{21}^{2} & r m_{12}^{2} & r m_{01}^{2} \\
-r m_{32}^{2 *} & -r m_{21}^{2 *} & -r m_{12}^{2 *} & -r m_{01}^{2 *}
\end{array}\right|}
$$

where $\tilde{\lambda}_{2}=-\tilde{\lambda}_{1}^{*}, \tilde{\lambda}_{4}=-\tilde{\lambda}_{3}^{*}$, and the corresponding solution of the Lax pair is $\left(-\varphi_{1}^{*} \phi_{1}^{*}\right)^{T}$, and

$$
r m_{j 1}^{l}=\frac{\partial^{l}}{\partial f^{l}}\left(\left(\widetilde{\lambda}_{0}+i f^{2}\right)^{j} \phi_{1}\left(\lambda_{0}+i f^{2}\right)\right), \quad r m_{j 2}^{l}=\frac{\partial^{l}}{\partial f^{l}}\left(\left(\widetilde{\lambda}_{0}+i f^{2}\right)^{j} \varphi_{1}\left(\lambda_{0}+i f^{2}\right)\right) .
$$

Similarly, the figures of three-order rogue wave solution can be given. Figure 5 is the threedimensional plot of $\left|q_{\mathrm{rw}}^{[3]}\right|$ with $r=1, a_{1}=0, c_{1}=1, \beta=1, \alpha=0$, and Figure 6 is the corresponding density plot.

\section{Modulation instability}

In order to discuss the modulation instability [50] of the mixed NLS equation (5), we first consider the steady state solution $q_{m 1}=a e^{i \omega t}$, where $\omega=a^{2}+r$. Then putting a small perturbation $U_{1}$ onto the steady state solution, we have $q_{m 2}=\left(a+U_{1}\right) e^{i \omega t}$. 


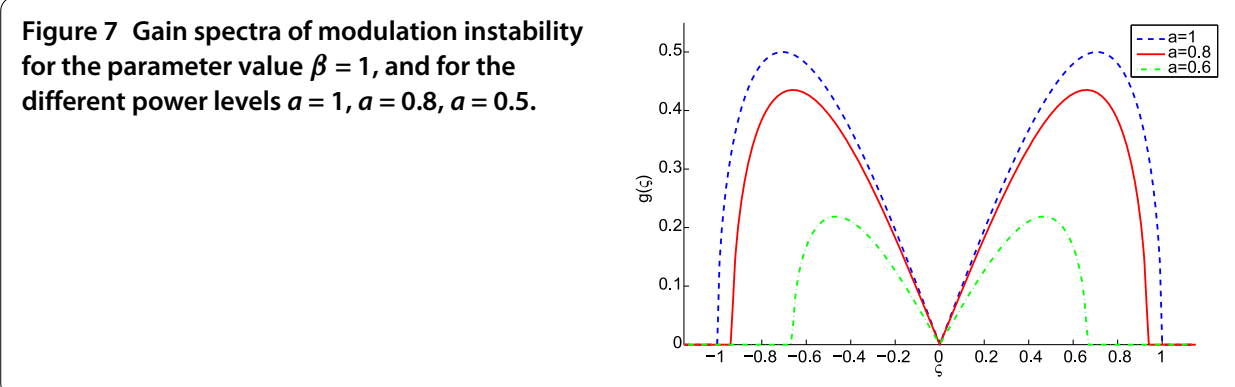

After the simple substitution and linearizing the equation, we have

$$
i U_{1 t}+U_{1 x x}+i \beta a^{2}\left(2 U_{1 x}+\widetilde{U}_{1 x}\right)+i \alpha U_{1 x}+a^{2}\left(U_{1}+\bar{U}_{1}\right)=0
$$

Immediately following this we set

$$
U_{1}=\mu \cos (\varsigma x+\tau t)+i v \sin (\varsigma x+\tau t)
$$

where $\varsigma$ is the frequency and $\tau$ is the wave number of the perturbation. Substituting the assumed solution into the linearized equation, we have a split of real and imaginary parts, namely

$$
\begin{aligned}
& \mu\left(-\varsigma^{2}+2 a^{2}\right)+v\left(-\tau-a^{2} \beta \varsigma-\alpha \varsigma\right)=0, \\
& \mu\left(-\tau-3 a^{2} \beta \varsigma-\alpha \varsigma\right)+v\left(-\varsigma^{2}\right)=0 .
\end{aligned}
$$

According to the initial condition we have the following determinant:

$$
\left(\tau+a^{2} \beta \varsigma\right)\left(\tau+3 a^{2} \beta \varsigma+\alpha \varsigma\right)-\varsigma^{2}\left(\varsigma^{2}-2 a^{2}\right)=0 .
$$

Solving the determinant we obtain the following dispersion relation:

$$
\tau=-2 a^{2}-\alpha \pm \varsigma \sqrt{\varsigma^{2}+a^{4} \beta^{2}-2 a^{2}} .
$$

Depending on the relation above, we know that if $\varsigma^{2}+a^{4} \beta^{2}-2 a^{2}>0$ the frequency $\varsigma$ is real at any value of the wave number $\tau$, whereas $\varsigma$ becomes complex. That is to say, in the case of $\varsigma^{2}+a^{4} \beta^{2}-2 a^{2}<0$ the instability region of the disturbance will grow with time exponentially. Then we consider the gain spectrum of MI. According to the above result, we have

$$
g(\varsigma)=2 \operatorname{Im}(\tau)=2 \sqrt{\left(\varsigma^{2}+a^{4} \beta^{2}-2 a^{2}\right) \varsigma^{2}},
$$

where $g(\varsigma)$ represents the gain of the frequency $\varsigma$. Figure 7 shows the gain at three different power levels.

\section{Conclusions}

In conclusion, we have constructed the one-, two-, and three-order rogue wave solutions for the general mixed NLS equation (5) by using the generalized DT. The $n$-fold DT is 
given by a gauge transformation, then a generalized DT is proposed through the Taylor expansion and a limit procedure. Moreover, some exact rogue wave solutions are derived explicitly. What is more, it is found from the plots of the rogue waves that the rogue waves appear suddenly and disappear quickly in the space-time framework. Finally, we give evidence for the connection between the occurrence of the rogue wave solution and the modulation instability.

\section{Appendix 1}

In this paper, we use the $T_{n}$ when $n$ is even [41]; $\left.T_{n}\right|_{n=2 k}(k=1,2,3, \ldots)$ can be expressed as follows:

$$
T_{n}=\left(\begin{array}{ll}
\frac{T_{11}}{\Delta_{1}} & \frac{T_{12}}{\Delta_{1}} \\
\frac{T_{13}}{\Delta_{2}} & \frac{T_{14}}{\Delta_{2}}
\end{array}\right),
$$

where

$$
\begin{aligned}
& T_{11}=\left|\begin{array}{cccccc}
\tilde{\lambda}^{n} & 0 & \ldots & \widetilde{\lambda}^{2} & 0 & 1 \\
\tilde{\lambda}_{1}^{n} \phi_{1} & \tilde{\lambda}_{1}^{n-1} \varphi_{1} & \ldots & \widetilde{\lambda}_{1}^{2} \phi_{1} & \widetilde{\lambda}_{1} \varphi_{1} & \phi_{1} \\
\tilde{\lambda}_{2}^{n} \phi_{2} & \widetilde{\lambda}_{2}^{n-1} \varphi_{2} & \ldots & \widetilde{\lambda}_{2}^{2} \phi_{2} & \tilde{\lambda}_{2} \varphi_{2} & \phi_{2} \\
\vdots & \vdots & \vdots & \vdots & \vdots & \vdots \\
\tilde{\lambda}_{n-1}^{n} \phi_{n-1} & \tilde{\lambda}_{n-1}^{n-1} \varphi_{n-1} & \ldots & \tilde{\lambda}_{n-1}^{2} \phi_{n-1} & \tilde{\lambda}_{n-1} \varphi_{n-1} & \phi_{n-1} \\
\tilde{\lambda}_{n}^{n} \phi_{n} & \tilde{\lambda}_{n}^{n-1} \varphi_{n} & \ldots & \widetilde{\lambda}_{n}^{2} \phi_{n} & \tilde{\lambda}_{n} \varphi_{n} & \phi_{n}
\end{array}\right|, \\
& T_{12}=\left|\begin{array}{cccccc}
0 & \tilde{\lambda}^{n-1} & \ldots & 0 & \tilde{\lambda} & 0 \\
\tilde{\lambda}_{1}^{n} \phi_{1} & \tilde{\lambda}_{1}^{n-1} \varphi_{1} & \ldots & \tilde{\lambda}_{1}^{2} \phi_{1} & \tilde{\lambda}_{1} \varphi_{1} & \phi_{1} \\
\tilde{\lambda}_{2}^{n} \phi_{2} & \tilde{\lambda}_{2}^{n-1} \varphi_{2} & \ldots & \widetilde{\lambda}_{2}^{2} \phi_{2} & \tilde{\lambda}_{2} \varphi_{2} & \phi_{2} \\
\vdots & \vdots & \vdots & \vdots & \vdots & \vdots \\
\tilde{\lambda}_{n-1}^{n} \phi_{n-1} & \tilde{\lambda}_{n-1}^{n-1} \varphi_{n-1} & \ldots & \tilde{\lambda}_{n-1}^{2} \phi_{n-1} & \tilde{\lambda}_{n-1} \varphi_{n-1} & \phi_{n-1} \\
\tilde{\lambda}_{n}^{n} \phi_{n} & \tilde{\lambda}_{n}^{n-1} \varphi_{n} & \ldots & \tilde{\lambda}_{n}^{2} \phi_{n} & \tilde{\lambda}_{n} \varphi_{n} & \phi_{n}
\end{array}\right|, \\
& T_{21}=\left|\begin{array}{cccccc}
0 & \tilde{\lambda}^{n-1} & \ldots & 0 & \tilde{\lambda} & 0 \\
\tilde{\lambda}_{1}^{n} \varphi_{1} & \tilde{\lambda}_{1}^{n-1} \phi_{1} & \ldots & \tilde{\lambda}_{1}^{2} \varphi_{1} & \tilde{\lambda}_{1} \phi_{1} & \varphi_{1} \\
\tilde{\lambda}_{2}^{n} \varphi_{2} & \tilde{\lambda}_{2}^{n-1} \phi_{2} & \ldots & \widetilde{\lambda}_{2}^{2} \varphi_{2} & \tilde{\lambda}_{2} \phi_{2} & \varphi_{2} \\
\vdots & \vdots & \vdots & \vdots & \vdots & \vdots \\
\tilde{\lambda}_{n-1}^{n} \varphi_{n-1} & \tilde{\lambda}_{n-1}^{n-1} \phi_{n-1} & \ldots & \tilde{\lambda}_{n-1}^{2} \varphi_{n-1} & \tilde{\lambda}_{n-1} \phi_{n-1} & \varphi_{n-1} \\
\tilde{\lambda}_{n}^{n} \varphi_{n} & \tilde{\lambda}_{n}^{n-1} \phi_{n} & \ldots & \tilde{\lambda}_{n}^{2} \varphi_{n} & \tilde{\lambda}_{n} \phi_{n} & \varphi_{n}
\end{array}\right|, \\
& T_{22}=\left|\begin{array}{cccccc}
\tilde{\lambda}^{n} & 0 & \ldots & \tilde{\lambda}^{2} & 0 & 1 \\
\tilde{\lambda}_{1}^{n} \varphi & \tilde{\lambda}_{1}^{n-1} \phi_{1} & \ldots & \tilde{\lambda}_{1}^{2} \varphi_{1} & \tilde{\lambda}_{1} \phi_{1} & \varphi_{1} \\
\tilde{\lambda}_{2}^{n} \varphi_{2} & \tilde{\lambda}_{2}^{n-1} \phi_{2} & \ldots & \tilde{\lambda}_{2}^{2} \varphi_{2} & \tilde{\lambda}_{2} \phi_{2} & \varphi_{2} \\
\vdots & \vdots & \vdots & \vdots & \vdots & \vdots \\
\tilde{\lambda}_{n-1}^{n} \varphi_{n-1} & \tilde{\lambda}_{n-1}^{n-1} \phi_{n-1} & \ldots & \tilde{\lambda}_{n-1}^{2} \varphi_{n-1} & \tilde{\lambda}_{n-1} \phi_{n-1} & \varphi_{n-1} \\
\tilde{\lambda}_{n}^{n} \varphi_{n} & \tilde{\lambda}_{n}^{n-1} \phi_{n} & \ldots & \tilde{\lambda}_{n}^{2} \varphi_{n} & \tilde{\lambda}_{n} \phi_{n} & \varphi_{n}
\end{array}\right|,
\end{aligned}
$$




$$
\begin{aligned}
\Delta_{1} & =\left|\begin{array}{ccccc}
\tilde{\lambda}_{1}^{n-1} \phi_{1} & \tilde{\lambda}_{1}^{n-2} \varphi_{1} & \ldots & \tilde{\lambda}_{1} \phi_{1} & \varphi_{1} \\
\tilde{\lambda}_{2}^{n-1} \phi_{2} & \tilde{\lambda}_{2}^{n-2} \varphi_{2} & \ldots & \tilde{\lambda}_{2} \phi_{2} & \varphi_{2} \\
\vdots & \vdots & \vdots & \vdots & \vdots \\
\tilde{\lambda}_{n-1}^{n-1} \phi_{n-1} & \tilde{\lambda}_{n-1}^{n-2} \varphi_{n-1} & \ldots & \tilde{\lambda}_{n-1} \phi_{n-1} & \varphi_{n-1} \\
\tilde{\lambda}_{n}^{n-1} \phi_{n} & \tilde{\lambda}_{n}^{n-2} \varphi_{n} & \ldots & \tilde{\lambda}_{n} \phi_{n} & \varphi_{n}
\end{array}\right|, \\
\Delta_{2} & =\left|\begin{array}{ccccc}
\tilde{\lambda}_{1}^{n-1} \varphi_{1} & \tilde{\lambda}_{1}^{n-2} \phi_{1} & \ldots & \tilde{\lambda}_{1} \varphi_{1} & \phi_{1} \\
\tilde{\lambda}_{2}^{n-1} \varphi_{2} & \tilde{\lambda}_{2}^{n-2} \phi_{2} & \ldots & \tilde{\lambda}_{2} \varphi_{1} & \phi_{2} \\
\vdots & \vdots & \vdots & \vdots & \vdots \\
\tilde{\lambda}_{n-1}^{n-1} \varphi_{2} & \tilde{\lambda}_{n-1}^{n-2} \phi_{n-1} & \ldots & \tilde{\lambda}_{n-1} \varphi_{1} & \phi_{n-1} \\
\tilde{\lambda}_{n}^{n-1} \varphi_{2} & \tilde{\lambda}_{n}^{n-2} \phi_{n} & \ldots & \tilde{\lambda}_{n} \varphi_{1} & \phi_{n}
\end{array}\right|
\end{aligned}
$$

\section{Appendix 2}

The rational solution of a two-order rogue wave is

$$
\begin{aligned}
& G_{3}=-162 t+54 x-162 i t-360 t x+72 x^{2}+432 t^{2}+1,152 t^{2} x-504 t x^{2}+72 x^{3} \\
& -864 t^{3}+60 x^{4}+2,052 t^{4}-3,120 t^{3} x+1,872 t^{2} x^{2}-528 t x^{3}+24 x^{5}-2,196 t^{5} \\
& -276 x^{4} t+4,104 t^{4} x-3,192 t^{3} x^{2}+1,296 t^{2} x^{3}+198 x^{5} t^{2}+2,050 t^{6} x-2,490 t^{5} x^{2} \\
& +1,734 t^{4} x^{3}-3,660 t^{5} x+3,624 t^{4} x^{2}-108 x^{5} t+642 x^{4} t^{2}-1,976 t^{3} x^{3}-30 x^{6} t \\
& +8 x^{6}+1,600 t^{6}-750 t^{7}+2 x^{7}+i x^{8}+54 i x+666 i t^{2}+90 i x^{2}-1,224 i t^{3}+72 i x^{3} \\
& +48 i x^{4}+1,752 i t^{4}+18 i x^{5}-1,314 i t^{5}+10 i x^{6}+1,490 i t^{6}-1,500 i t^{7}+4 i x^{7}+18 i \\
& +18+625 i t^{8}-432 i t x+1,224 i t^{2} x-504 i t x^{2}+1,512 i t^{2} x^{2}-2,448 i t^{3} x-432 i t x^{3} \\
& -2,148 i t^{3} x^{2}+2,541 i t^{4} x+948 i t^{2} x^{3}-1,492 i x^{4} t^{3}-210 i t x^{4}-132 i x^{5} t+726 i x^{4} t^{2} \\
& -3,492 i t^{5} x+3,678 i t^{4} x^{2}-2,152 i t^{3} x^{3}-60 i^{6} t+396 i x^{5} t^{2}+4,100 i t^{6} x \\
& -4,980 i t^{5} x^{2}+3,468 i t^{4} x^{3}+1,366 i x^{4} t^{4}-496 i x^{5} t^{3}+116 i x^{6} t^{2} \\
& -16 i x^{7} t-16 i x^{7} t-2,000 i t^{7} x+2,900 i t^{6} x^{2}-2,480 i t^{5} x^{3}, \\
& G_{4}=-288 t-25,002 i t^{4} x-6,768 i t^{2} x^{2}+36 i+15,828 i t^{3} x^{2}-864 x t+36 x^{2}+2,340 t^{2} \\
& -2,256 i x^{4} t^{2}+5,940 t^{2} x,+72 x^{3}-7,872 t^{3}+78 x^{4}+13,950 t^{4}-15,816 t^{3} x \\
& +6,732 t^{2} x^{2}-1,224 t x^{3}+42 x^{5}-15,006 t^{5}-654 x^{4} t+22,410 t^{4} x-13,596 t^{3} x^{2} \\
& +4,164 t^{2} x^{3}-1,638 x^{5} t^{2}-15,602 t^{6} x+21,030 t^{5} x^{2}+30,520 t^{7} x-43,628 t^{6} x^{2} \\
& +152 x^{7} t-1,324 x^{6} t^{2}-18,986 t^{4} x^{4}-15,438 t^{4} x^{3}+6,376 x^{5} t^{3}+6,622 x^{4} t^{3} \\
& -13,596 t^{5} x+9,552 t^{4} x^{2}-156 x^{5} t+888 x^{4} t^{2}-3,640 t^{3} x^{3}+210 x^{6} t+9,375 t^{9} \\
& -9,575 t^{8}-5 x^{9}-7 x^{8}+16 x^{6}+7,976 t^{6}+4,890 t^{7}-10 x^{7}-3,125 t^{10}-x^{10} \\
& -120 i x^{7} t+1,260 i t^{6} x^{3}-24,420 i t^{6} x-144 i x^{2}+300 i t^{2} x^{7}+324 i x^{5} t+1,848 i t x^{3} \\
& -33,125 t^{8} x+53,500 t^{7} x^{2}-51,700 t^{6} x^{3}+32,890 t^{5} x^{4}-14,270 t^{4} x^{5}+4,220 t^{3} x^{6} \\
& -820 t^{2} x^{7}+95 t x^{8}+1,040 t^{3} x^{7}+12,500 t^{9} x+660 i x^{6} t^{2}+13,800 i t^{7} x \\
& -23,125 t^{8} x^{2}+2,600 t^{7} x^{3}-19,650 t^{6} x^{4}+10,424 t^{5} x^{5}-393-t^{4} x^{6}-185 t^{2} x^{8}
\end{aligned}
$$




$$
\begin{aligned}
+ & 20 t x^{9}+32,580 i t^{5} x-23,736 i t^{4} x^{2}+9,480 i x^{3} t^{3}-3,342 i t^{5} x^{4}-144 i t-432 i t^{2} \\
+ & 1,656 i t x^{2}-5,676 i t^{2} x^{3}+1,182 i t x^{4}-144 i x^{4} t^{3}+1,830 i x^{4} t^{4}-1,800 i x^{5} t^{3} \\
& -144 i x^{6} t+564 i x^{5} t^{2}+17,808 i t^{5} x^{2}-5,436 i t^{4} x^{3}+12,984 i t^{3} x-11,340 i t^{6} x^{2} \\
+ & 2,904 i t^{4} x^{3}-4,125 i t^{8} x+2,700 i t^{7} x^{2}+2,610 i t^{4} x^{5}-1,140 i t^{3} x^{6}-45 i t x^{8} \\
+ & 504 i t x-4,032 i t^{2} x+3,984 i t^{3}-264 i x^{3}-216 i x^{4}-10,968 i t^{4}-1,368 t x^{2} \\
& -114 i x^{5}+17,478 i t^{5}-24 i x^{6}-19,200 i t^{6}+13,200 i t^{7}+12 i x^{7} \\
+ & 9 i x^{8}-6,375 i t^{8}+1,875 i t^{9}+3 i x^{9}+36,232 t^{5} x, \\
H_{1}= & 162 t-54 x-162 i t+360 x t-72 x^{2}-432 t^{2}-1,152 t^{2} x+504 t x^{2}-72 x^{3}+864 t^{3} \\
- & 60 x^{4}-2,052 t^{4}+3,120 x t^{3}-18-1,872 x^{2} t^{2}+528 x^{3} t-24 x^{5}+2,196 t^{5} \\
+ & 276 x^{4} t-4,104 x t^{4}+3,192 x^{2} t^{3}-1,296 x^{3} t^{2}-198 x^{5} t^{2}-2,050 x t^{6}+2,490 x^{2} t^{5} \\
& -1,734 x^{3} t^{4}+746 x^{4} t^{3}+3,660 x t^{5}-3,624 x^{2} t^{4}+108 x^{5} t-624 x^{4} t^{2}+1,976 x^{3} t^{3} \\
+ & 30 x^{6} t-8 x^{6}-1,600 t^{6}+750 t^{7}+18 i-2 x^{7}+i x^{8}+54 i x+666 i t^{2}+90 i x^{2} \\
& -1,224 i t^{3}+72 i x^{3}+48 i x^{4}+1,752 i t^{4}+18 i x^{5}-1,314 i t^{5}+10 i x^{6}+1,490 i x^{6} \\
& -1,500 i t^{7}+4 i x^{7}+625 i t^{8}-432 i x t+1,224 i x t^{2}-504 i x^{2} t+1,512 i x^{2} t^{2} \\
& -2,448 i x t^{3}-432 i x^{3} t-2,148 i x^{2} t^{3}+2,541 i x t^{4}+948 i x^{3} t^{3}-210 i x^{4} t \\
- & 132 i x^{5} t+726 i x^{5} t^{2}-3,492 i t^{5} x+3,678 i x^{2} t^{4}-2,125 i t^{3} x^{3}-1,492 i x^{4} t^{3} \\
& -60 i x^{6} t+396 i x^{5} t^{2}+4,100 i x t^{6}-4,980 i x^{2} t^{5}+3,468 i x^{3} t^{4}+1,336 i x^{4} t^{4} \\
& 496 i x^{5} t^{3}+116 i x^{6} t^{2}-16 i x^{7} t-2,000 i t^{7} x+2,900 i x^{2} t^{6}-2,480 i x^{3} t^{6} \\
& \\
&
\end{aligned}
$$

\section{Competing interests}

The authors declare that they have no competing interests.

\section{Authors' contributions}

All authors have made equal contributions. All authors have read and approved the final manuscript.

\section{Acknowledgements}

This work is supported by National Natural Science Foundation of China under Grant Nos. 11271362, 11201501, 11375030 and 11571389, Beijing Finance Funds of Natural Science Program for Excellent Talents No. 2014000026833ZK19, the Doctor Startup Fund of Liaoning Province (201501164) and the Fundamental Research Funds for the Central Universities under the number of DC201501040.

Received: 12 April 2016 Accepted: 8 August 2016 Published online: 05 September 2016

\section{References}

1. Ablowitz, MJ, Segur, H: Solitons and the Inverse Scattering Transform. SIAM, Philadelphia (1981)

2. Matveev, VB, Salle, MA: Darboux Transformations and Solitons. Springer, Berlin (1991)

3. Wang, DS, Chen, F, Wen, XY: Darboux transformation of the general Hirota equation: multisoliton solutions, breather solutions, and rogue wave solutions. Adv. Differ. Equ. 2016, 67 (2016). doi:10.1186/s13662-016-0780-z

4. Vekslerchik, VE: Backlund transformations for the Nizhnik-Novikov-Veselov equation. J. Phys. A 37, 5667-5678 (2004)

5. Wang, DS, Wei, X: Integrability and exact solutions of a two-component Korteweg-de Vries system. Appl. Math. Lett. 51, 60-67 (2016)

6. Wang, DS, Liu, J, Zhang, ZF: Integrability and equivalence relationships of six integrable coupled Korteweg-de Vries equations. Math. Methods Appl. Sci. 39, 3516-3530 (2016)

7. Wang, DS, Zhang, DJ, Yang, JK: Integrable properties of the general coupled nonlinear Schrödinger equations. J. Math. Phys. 51, $023510(2010)$

8. Wang, DS, Yin, SJ, Tian, Y, Liu, Y: Integrability and bright soliton solutions to the coupled nonlinear Schrodinger equation with higher-order effects. Appl. Math. Comput. 229, 296-309 (2014) 
9. Wang, DS, Ma, YQ, Li, XG: Prolongation structures and matter-wave solitons in $F=1$ spinor Bose-Einstein condensate with time-dependent atomic scattering lengths in an expulsive harmonic potential. Commun. Nonlinear Sci. Numer. Simul. 19, 3556-3569 (2014)

10. Hirota, R: Exact solution of the Korteweg de Vries equation for multiple collisions of solitons. Phys. Rev. Lett. 27, 1192-1194 (1971)

11. Wang, DS, Li, XG, Chan, CK, Zhou, J: Double Wronskian solution and soliton properties of the nonisospectral BKP equation. Commun. Theor. Phys. 65, 259-265 (2016)

12. Wang, DS, Yin, YB: Symmetry analysis and reductions of the two-dimensional generalized Benney system via geometric approach. Comput. Math. Appl. 71, 748-757 (2016)

13. Wang, DS, Hu, XH, Hu, J, Liu, WM: Quantized quasi-two-dimensional Bose-Einstein condensates with spatially modulated nonlinearity. Phys. Rev. A 81, 025604 (2010)

14. Wang, DS, Song, SW, Xiong, B, Liu, WM: Quantized vortices in a rotating Bose-Einstein condensate with spatiotemporally modulated interaction. Phys. Rev. A 84, 053607 (2011)

15. Wang, DS, Xue, YS, Zhang, ZF: Localized nonlinear matter waves in one-dimensional Bose-Einstein condensates with spatiotemporally modulated two- and three-body interactions. Rom. J. Phys. 61(5-6), 827-841 (2016)

16. Wang, DS, Shi, YR, Chow, KW, Yu, ZX, Li, XG: Matter-wave solitons in a spin-1 Bose-Einstein condensate with time-modulated external potential and scattering lengths. Eur. Phys. J. D 67, 242 (2013)

17. Ganji, DD, Rafei, M: Solitary wave solutions for a generalized Hirota-Satsuma coupled KdV equation by homotopy perturbation method. Phys. Lett. A 356, 131-137 (2006)

18. Kumar, D, Singh, J, Kumar, S, Sushila: Numerical computation of Klein-Gordon equations arising in quantum field theory by using homotopy analysis transform method. Alex. Eng. J. 53(2), 469-474 (2014)

19. Gupta, S, Kumar, D, Singh, J: Analytical solutions of convection-diffusion problems by combining Laplace transform method and homotopy perturbation method. Alex. Eng. J. 54(3), 645-651 (2015)

20. Ramswroop, Singh, J, Kumar, D: Numerical computation of fractional Lotka-Volterra equation arising in biological systems. Nonlinear Eng. 4(2), 117-125 (2015)

21. Wang, DS, Zhang, HQ: Further improved F-expansion method and new exact solutions of Konopelchenko-Dubrovsky equation. Chaos Solitons Fractals 25, 601-610 (2005)

22. Wang, DS, Li, XG: Localized nonlinear matter waves in a Bose-Einstein condensate with spatially inhomogeneous two- and three-body interactions. J. Phys. B, At. Mol. Opt. Phys. 45, 105301 (2012)

23. Wang, DS, Zeng, X, Ma, YQ: Exact vortex solitons in a quasi-two-dimensional Bose-Einstein condensate with spatially inhomogeneous cubic-quintic nonlinearity. Phys. Lett. A 376, 3067-3070 (2012)

24. Solli, DR, Ropers, C, Koonath, P, Jalali, B: Optical rogue waves. Nature 450, 1054-1058 (2007)

25. Dudley, JM, Genty, G, Dias, F, Kibler, B, Akhmediev, N: Modulation instability Akhmediev Breathers and continuous wave supercontinuum generation. Opt. Express 17, 21497-21508 (2009)

26. Genty, G, Dudley, JM, Eggleton, BJ: Modulation control and spectral shaping of optical fiber supercontinuum generation in the picosecond regime. Appl. Phys. B 94, 187-194 (2009)

27. Yeom, DI, Eggleton, BJ: Photonics: rogue wave surface in light. Nature 450, 953-954 (2007)

28. Ganshin, AN, Efimov, VB, Kolmakov, GV, Mezhov-Deglin, LP, McClintock, PVE: Observation of an inverse energy cascade in developed acoustic turbulence in superfluid helium. Phys. Rev. Lett. 101, 5498-5500 (2008)

29. Bludov, YV, Konotop, WV, Akhmediev, N: Matter rogue waves. Phys. Rev. A 80, 033610 (2009)

30. Wen, L, Li, L, Li, ZD, Song, SW, Zhang, XF, Liu, WM: Matter rogue wave in Bose-Einstein condensates with attractive atomic interaction. Eur. Phys. J. D 64, 473-478 (2011)

31. Wang, DS, Han, W, Shi, YR, Li, ZD, Liu, WM: Dynamics and stability of stationary states for the spin-1 Bose-Einstein condensates in a standing light wave. Commun. Nonlinear Sci. Numer. Simul. 36, 45-57 (2016)

32. Draper, L: 'Freak' ocean waves. Weather 21, 2-4 (1966)

33. Ramswroop, Singh, J, Kumar, D: Numerical study for time-fractional Schrödinger equations arising in quantum mechanics. Nonlinear Eng. 3(3), 169-177 (2014)

34. Kumar, D, Singh, J, Sushila: Application of homotopy perturbation transform method to linear and nonlinear Schrödinger equations. Int. J. Nonlinear Sci. 16, 203-209 (2013)

35. Eid, R, Muslih, SI, Baleanu, D, Rabeid, E: On fractional Schrödinger equation in $\alpha$-dimensional fractional space. Nonlinear Anal., Real World Appl. 10, 1299-1304 (2009)

36. Muslih, SI, Agrawal, OP, Baleanu, D: A fractional Schrödinger equation and its solution. Int. J. Theor. Phys. 49, 1746-1752 (2010)

37. Guo, BL, Ling, LM, Liu, QP: Nonlinear Schrödinger equation: generalized Darboux transformation and rogue wave solution. Phys. Rev. E 85, 026607 (2012)

38. Peregrine, DH: Water waves, nonlinear Schrödinger equations and their solutions. J. Aust. Math. Soc. Ser. B, Appl. Math 25, 16-43 (1983)

39. Zhao, QL: On Nth-order rogue wave solution to the generalized nonlinear Schrödinger equation. Phys. Rev. A 377 , 855-859 (2013)

40. Yang, B, Zhang, WG, Zhang, HQ, Pei, SB: Generalized Darboux transformation and rogue wave solutions for the higher-order dispersive nonlinear Schrödinger equation. Phys. Scr. 88, 065004 (2013)

41. He, J, Xu, S, Cheng, Y: The rational solutions of the mixed nonlinear Schrödinger equation. AIP Adv. 5, 603-634 (2015)

42. Wadati, M, Konno, K, Ichikawa, YH: A generalization of inverse scattering method. J. Phys. Soc. Jpn. 46, 1965-1966 (1997)

43. Rangwala, AA, Rao, JA: Backlund transformations, soliton solutions and wave functions of Kaup-Newell and Wadati-Konno-Ichikawa systems. J. Math. Phys. 31, 1126-1132 (1990)

44. Wrigh, OC: Homoclinic connections of unstable plane waves of the modified nonlinear Schrödinger equation. Chaos Solitons Fractals 20, 735-749 (2004)

45. Xia, T, Chen, X, Chen, D: Darboux transformation and soliton-like solutions of nonlinear Schrödinger equations. Chaos Solitons Fractals 26, 889-896 (2005)

46. Zhang, HQ, Zhai, BG, Wang, XL: Soliton and breather solutions of the modified nonlinear Schrödinger equation. Phys. Scr. 85, 015007 (2012) 
47. Liu, SL, Wang, WZ: Exact N-soliton solution of the modified nonlinear Schrödinger equation. Phys. Rev. E 48, 3054-3059 (1993)

48. Li, M, Tian, B, Liu, WJ, Zhang, HQ, Wang, P: Dark and antidark solitons in the modified nonlinear Schrödinger equation accounting for the self-steepening effect. Phys. Rev. E 81, 046606 (2010)

49. He, JS, Zhang, L, Cheng, Y, Li, YS: Determinant representation of Darboux transformation for the AKNS system. Sci. China Ser. A 49, 1867-1878 (2006)

50. Zhang, HQ, Tian, B, Meng, XH, Lv, X, Liu, WJ: Conservation laws, soliton solutions and modulational instability for the higher-order dispersive nonlinear Schrödinger equation. Eur. Phys. J. B 72, 233-239 (2009)

Submit your manuscript to a SpringerOpen ${ }^{\circ}$ journal and benefit from:

- Convenient online submission

Rigorous peer review

- Immediate publication on acceptance

- Open access: articles freely available online

- High visibility within the field

- Retaining the copyright to your article 